Supporting Information for

\title{
An Adamantane-based Coordination Framework with the First Observation of Discrete Metal Sulfonate Clusters
}

\author{
Dennis J. Hoffart, Adrien P. Côté, George K. H. Shimizu* \\ Department of Chemistry, University of Calgary, Calgary, Alberta, \\ T2N 1N4 Canada
}

Preparation of 1,3,5,7-Tetrakis(4-chlorosulfonylphenyl)adamantane: Tetraphenyl adamantane $^{1}(1.6390 \mathrm{~g}, 3.72 \mathrm{mmol})$ was suspended in $25 \mathrm{~mL}$ of dichloromethane and chlorosulfonic acid ( $2.5 \mathrm{~mL}, 37.61 \mathrm{mmol}, 10$ equiv.) was added dropwise over $5 \mathrm{~min}$. The mixture was heated to $35^{\circ} \mathrm{C}$ for $75 \mathrm{~min}$, and cooled to room temperature. After this time, a brown/black oil separates from the yellow dichloromethane solution, which was removed by centrifugation. The yellow supernatant was decanted and the solvent removed under reduced pressure to give a yellow oil. Water was layered over oil and the two phases were sonicated to evolve a white powder which was filtered off, washed with water $(2 \times 2 \mathrm{~mL})$, and dried to yield exclusively the tetrachlorosulfonated product. Yield: $2.73 \mathrm{~g}(85 \%)$. ${ }^{1} \mathrm{H}$ NMR $\left(200 \mathrm{MHz}, \mathrm{CD}_{2} \mathrm{Cl}_{2}\right): \delta(\mathrm{ppm})=8.08(\mathrm{~d}, 8 \mathrm{H}), 7.77(\mathrm{~d}, 8 \mathrm{H}), 2.29(\mathrm{~s}$, $12 \mathrm{H}) .{ }^{13} \mathrm{C} \mathrm{NMR}\left(50 \mathrm{MHz}, \mathrm{CD}_{2} \mathrm{Cl}_{2}\right): \delta(\mathrm{ppm})=156.2,142.9,127.8,127.8,127.0,46.3$, 40.4. FT-IR (KBr, cm $\left.{ }^{-1}\right)$ : $3403(\mathrm{br}), 2957(\mathrm{~m}), 1591(\mathrm{~m}), 1524(\mathrm{~m}), 1373(\mathrm{~s}), 1169(\mathrm{~s})$, 1084 (w) 833 (w), 705 (s), 585 (s). EA (C,H) for $\mathrm{C}_{34} \mathrm{H}_{28} \mathrm{~S}_{4} \mathrm{O}_{8} \mathrm{Cl}_{4}$ : Anal. calcd.: C, 49.04; $\mathrm{H}$, 3.39. Found: C, 49.59; H, 2.96. 
Preparation of 1,3,5,7-Tetrakis(4-sulfophenyl)adamantane: 1,3,5,7-Tetrakis-(4sulfonylchlorophenyl) adamantane $(2.72 \mathrm{~g}, 3.28 \mathrm{mmol})$ was suspended in $750 \mathrm{~mL}$ of water and heated to reflux for $48 \mathrm{~h}$ to give a clear solution. Solvent was removed under reduced pressure and the resulting white solid heated $\left(40^{\circ} \mathrm{C}\right)$ under dynamic vacuum for $5 \mathrm{~h}$ to assure complete removal of any residual $\mathrm{HCl}$. Yield: $2.49 \mathrm{~g}(90 \%)$. ${ }^{1} \mathrm{H}$ NMR (200 $\left.\mathrm{MHz}, \mathrm{MeOH}-d_{4}\right): \delta(p p m)=7.80(\mathrm{~d}, 8 \mathrm{H}), 7.66(\mathrm{~d}, 8 \mathrm{H}), 2.23(\mathrm{~s}, 12 \mathrm{H}) .{ }^{13} \mathrm{C}$ NMR $(50 \mathrm{MHz}$, $\left.\mathrm{D}_{2} \mathrm{O}\right): \delta(\mathrm{ppm})=153.2,140.4,126.0,125.7,45.9,39.5 . \mathrm{FT}-\mathrm{IR}\left(\mathrm{KBr}, \mathrm{cm}^{-1}\right): 3983(\mathrm{br})$, $2957(\mathrm{~m}), 1599$ (w), $1496(\mathrm{w}), 1445$ (w), 1399 (w), 1231 (s, br), 1171 (s, br) 1128 (s, br), 1033 (s), $833(w), 714(\mathrm{~m})$. ESI-MS (neg. ion, $\mathrm{H}_{2} \mathrm{O} ; M=: 752.81$ for $\mathrm{C}_{34} \mathrm{H}_{24} \mathrm{~S}_{4} \mathrm{O}_{12}$ ): $\mathrm{m} / \mathrm{z}$ $(\mathrm{amu})=M\left(\mathrm{H}_{2} \mathrm{O}\right)_{12}, 987.40 ; M\left(\mathrm{H}_{2} \mathrm{O}\right)_{25}$, 1203.02. EA $(\mathrm{C}, \mathrm{H})$ for $\mathrm{C}_{34} \mathrm{H}_{28} \mathrm{~S}_{4} \mathrm{O}_{12}\left(\mathrm{H}_{2} \mathrm{O}\right)_{11}$ : Anal. calcd.: C, 42.76; $\mathrm{H}, 5.28$. Found: $\mathrm{C}, 42.83 ; \mathrm{H}, 5.31$. TGA/DSC: $30-115{ }^{\circ} \mathrm{C}$ (DSC featureless) $-13.8 \%$ obs. $14.2 .0 \%$ calcd. for loss of $8 \mathrm{H}_{2} \mathrm{O} ; 215{ }^{\circ} \mathrm{C}$ decomposition.

1. For the preparation of tetraphenyl adamantane see: Stetter, H.; Krause, M. Liebigs Ann. 1968, 717, 60.

Preparation of $\left[\mathrm{Ba}_{2}(\mathrm{~L})\left(\mathrm{H}_{2} \mathrm{O}\right)_{4}(\right.$ dioxane $\left.)\right] \cdot\left(\mathrm{H}_{2} \mathrm{O}\right)_{3}(\text { dioxane })_{3}, \mathbf{1}: \mathrm{H}_{4} \mathbf{L}(150 \mathrm{mg}, 0.20 \mathrm{mmol})$ was dissolved in $\mathrm{H}_{2} \mathrm{O}(5 \mathrm{~mL})$ to which $\mathrm{Ba}(\mathrm{OH})_{2}(68 \mathrm{mg}, 0.40 \mathrm{mmol})$ was added to give a turbid solution. The mixture was stirred (12 h) at ambient temperature, filtered, and dioxane vapor diffused into this solution to yield X-ray quality crystals. Slow vapor diffusion of dioxane into the aqueous solution gave colorless plates of 1 suitable for X-ray data collection. Elemental analysis was performed on a sample air dried at $25^{\circ} \mathrm{C}$ for $1 \mathrm{~h}$. Found: C, $36.45 \% ; \mathrm{H}, 4.01 \%$. Calcd for $\left[\mathrm{Ba}_{2}(\mathrm{~L})\left(\mathrm{H}_{2} \mathrm{O}\right)_{4}(\right.$ dioxane $\left.)\right] \bullet\left(\mathrm{H}_{2} \mathrm{O}\right)_{3}: \mathrm{C}, 36.19 \% ; \mathrm{H}$, 3.84 \%. IR $\left(\mathrm{cm}^{-1}\right): 3430.0,2924.9,2898.1,2853.0,1633.2,1599.6,1497.3,1399.1$, 1175.7, 1127.7, 10337.0, 1007.7716.2, 623.3. 
Figure S1: Elemental Analysis for 1,3,5,7-Tetrakis(4-chlorosulfonylphenyl)adamantane

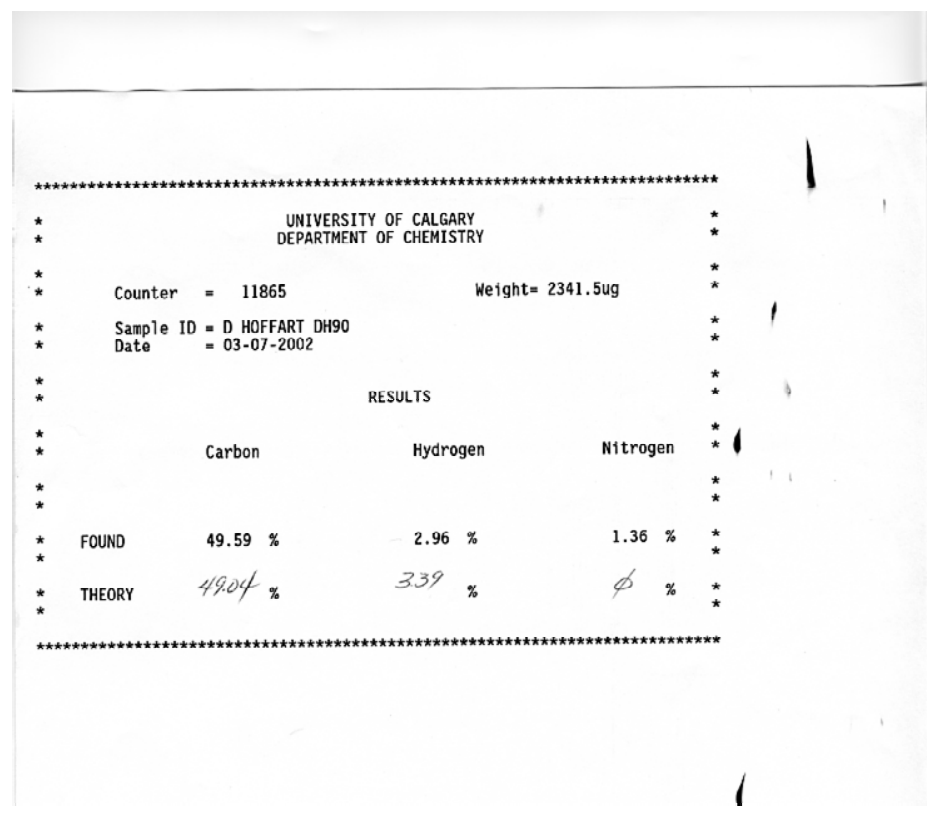

Figure S2: ${ }^{1} \mathrm{H}$ NMR for 1,3,5,7-Tetrakis(4-chlorosulfonylphenyl)adamantane

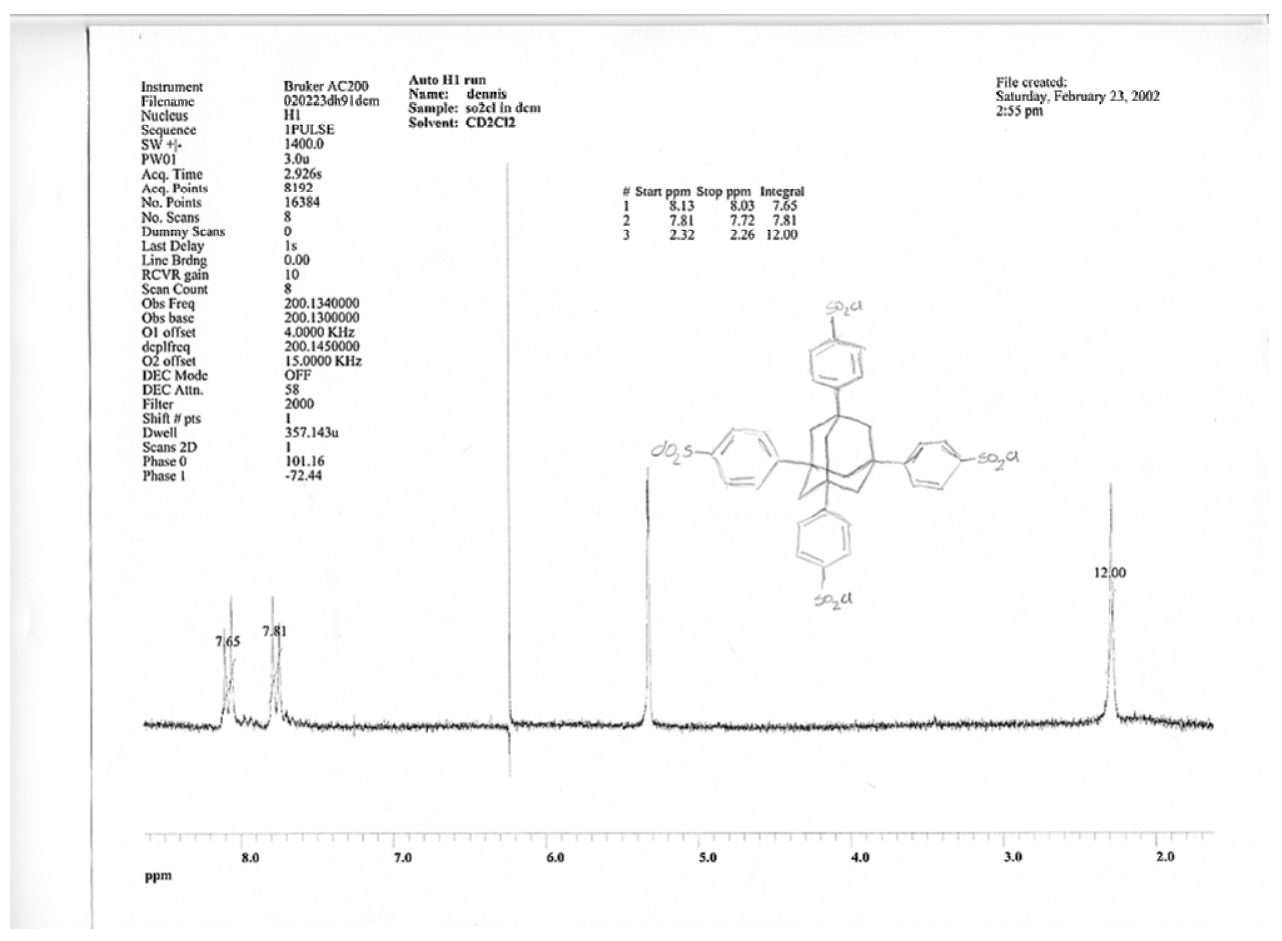


Figure S3: ${ }^{13} \mathrm{C}$ NMR for 1,3,5,7-Tetrakis(4-chlorosulfonylphenyl)adamantane

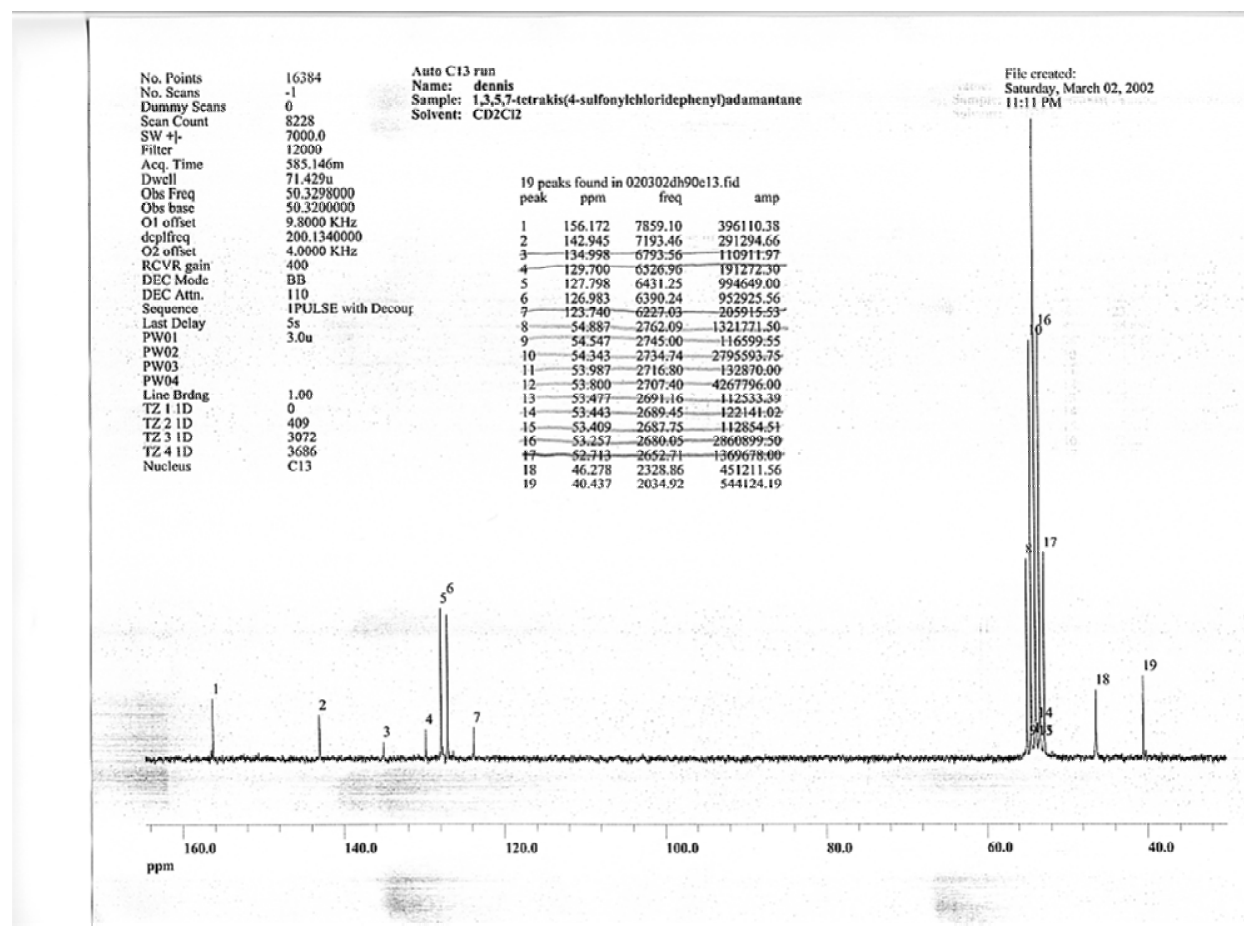

Figure S4: Elemental Analysis for 1,3,5,7-Tetrakis(4-sulfophenyl)adamantane

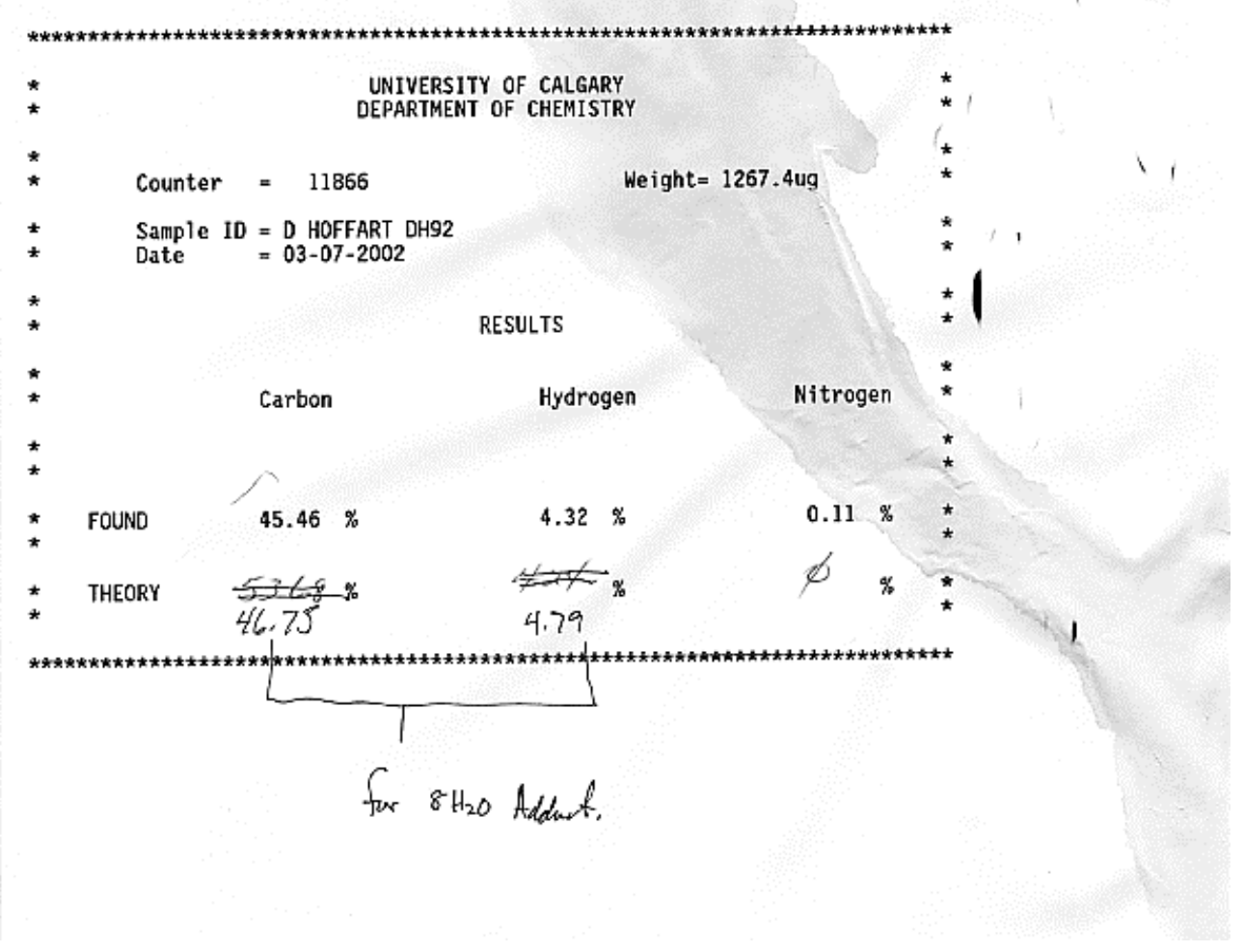


Figure S5: ${ }^{1} \mathrm{H}$ NMR for 1,3,5,7-Tetrakis(4-sulfophenyl)adamantane

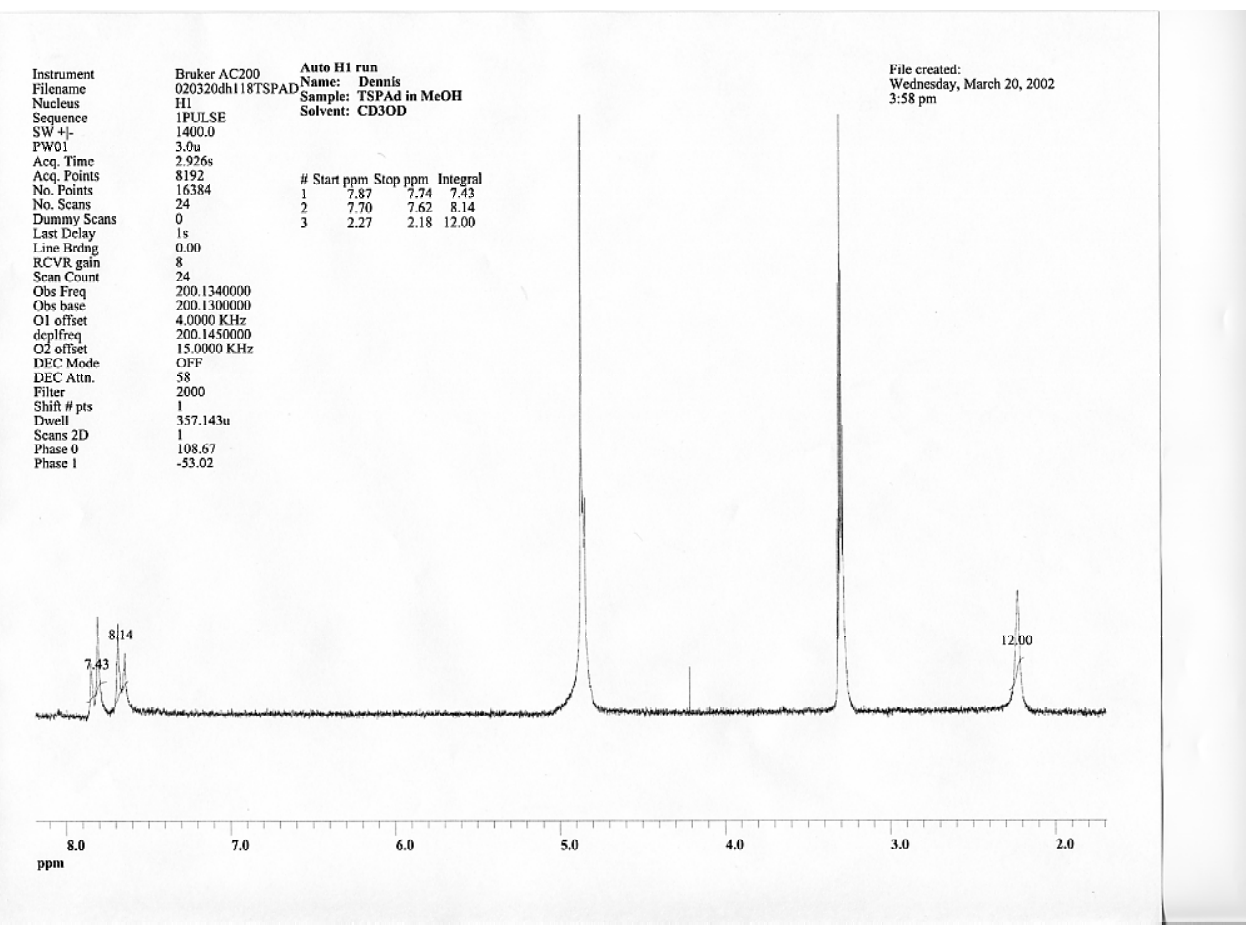

Figure S6: ${ }^{13} \mathrm{C}$ NMR for 1,3,5,7-Tetrakis(4-sulfophenyl)adamantane 


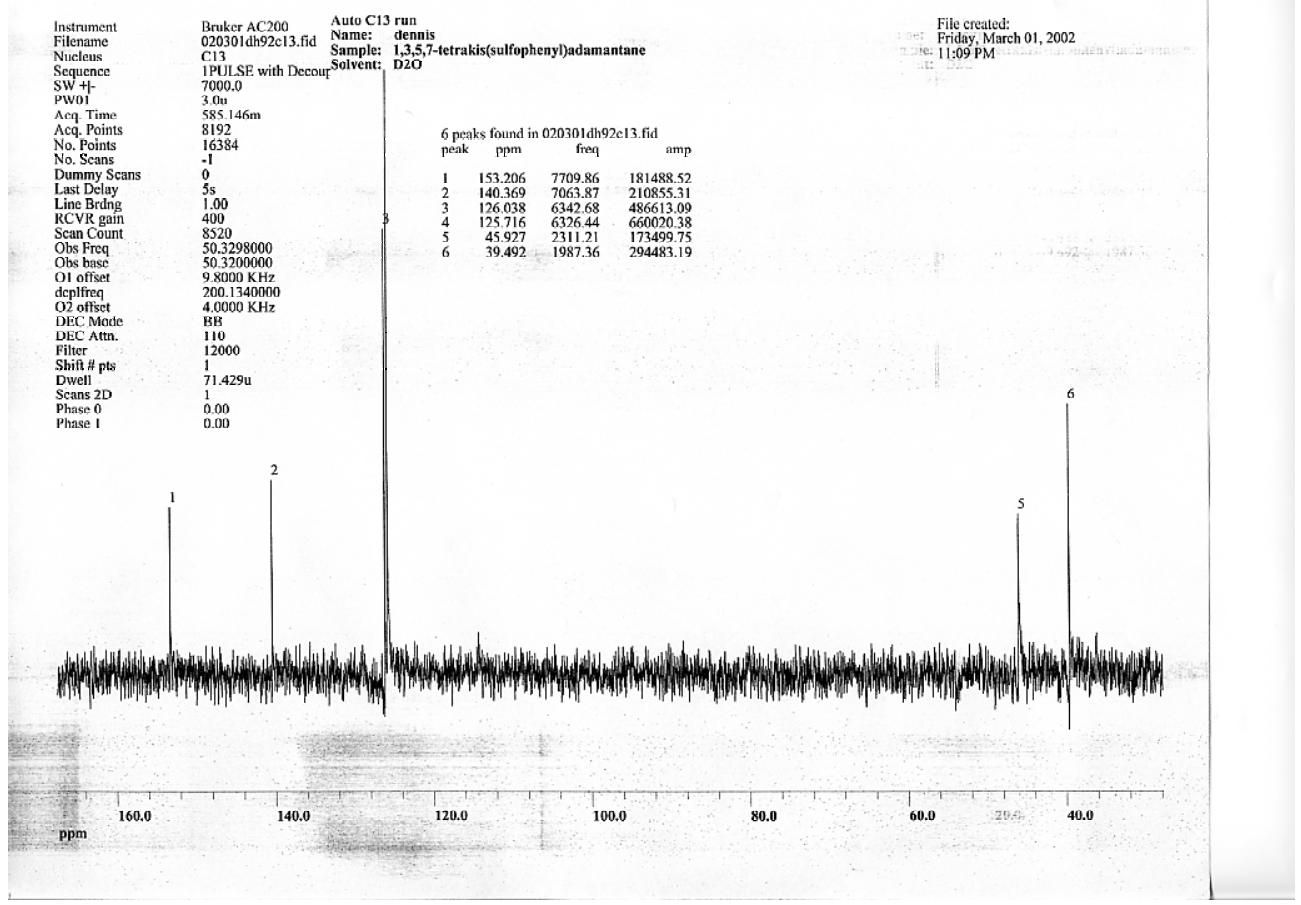


Figure S7: ESI-MS for 1,3,5,7-Tetrakis(4-sulfophenyl)adamantane

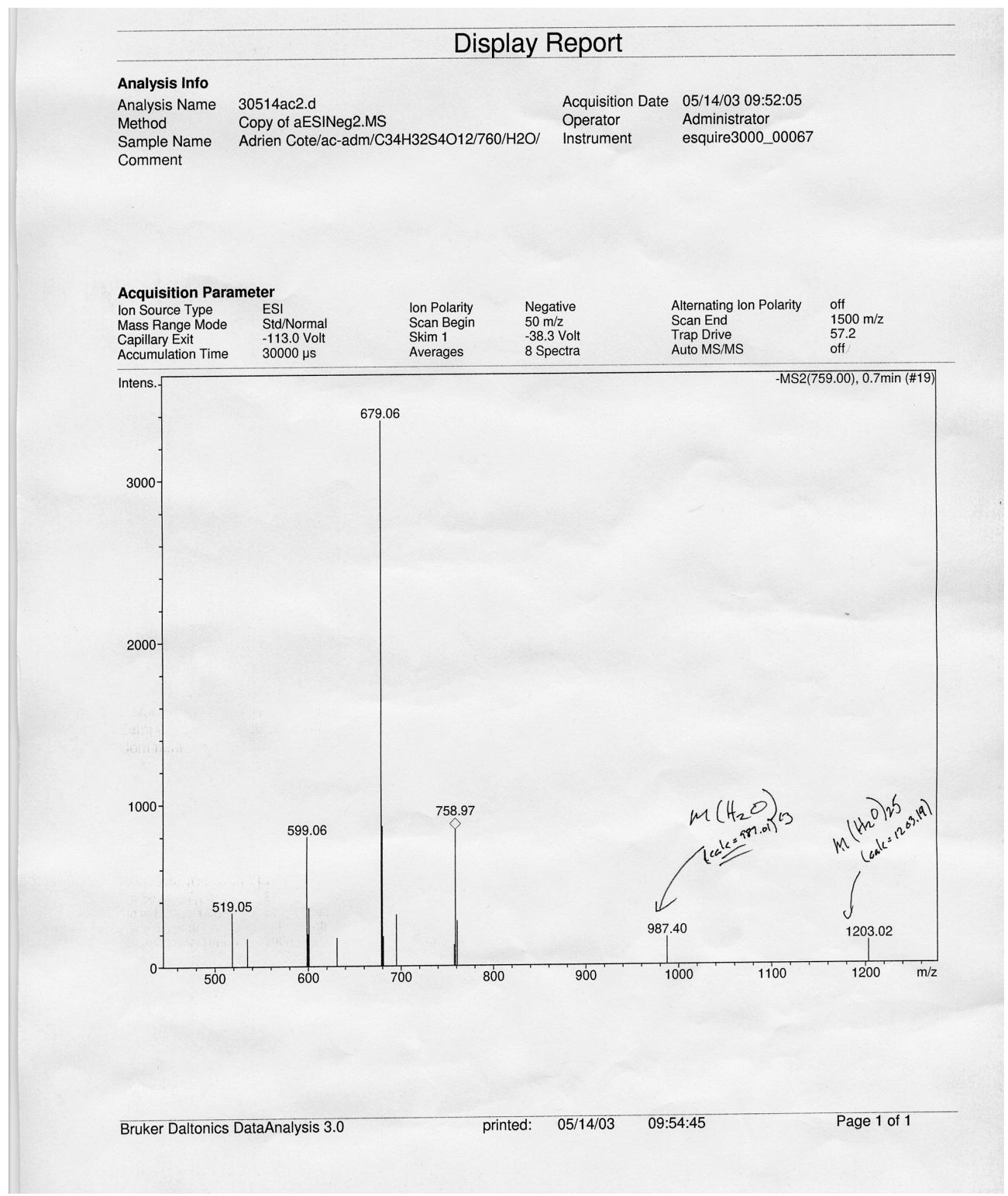


Figure S8: TGA/DSC for 1,3,5,7-Tetrakis(4-sulfophenyl)adamantane

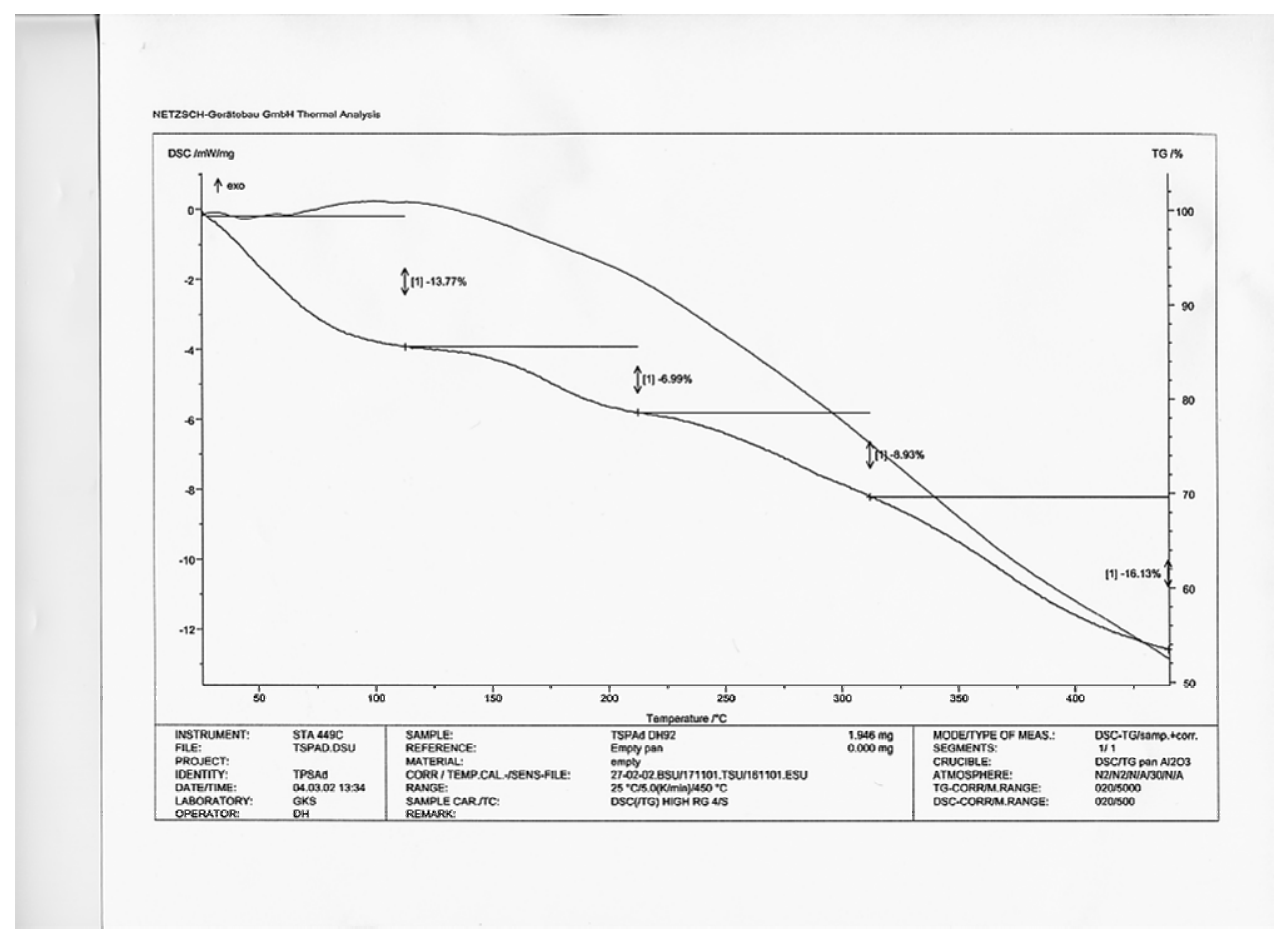

Figure S9: TGA/DSC for $\left[\mathrm{Ba}_{2}(\mathrm{~L})\left(\mathrm{H}_{2} \mathrm{O}\right)_{4}(\right.$ dioxane $\left.)\right] \cdot\left(\mathrm{H}_{2} \mathrm{O}\right)_{3}(\text { dioxane })_{3}$

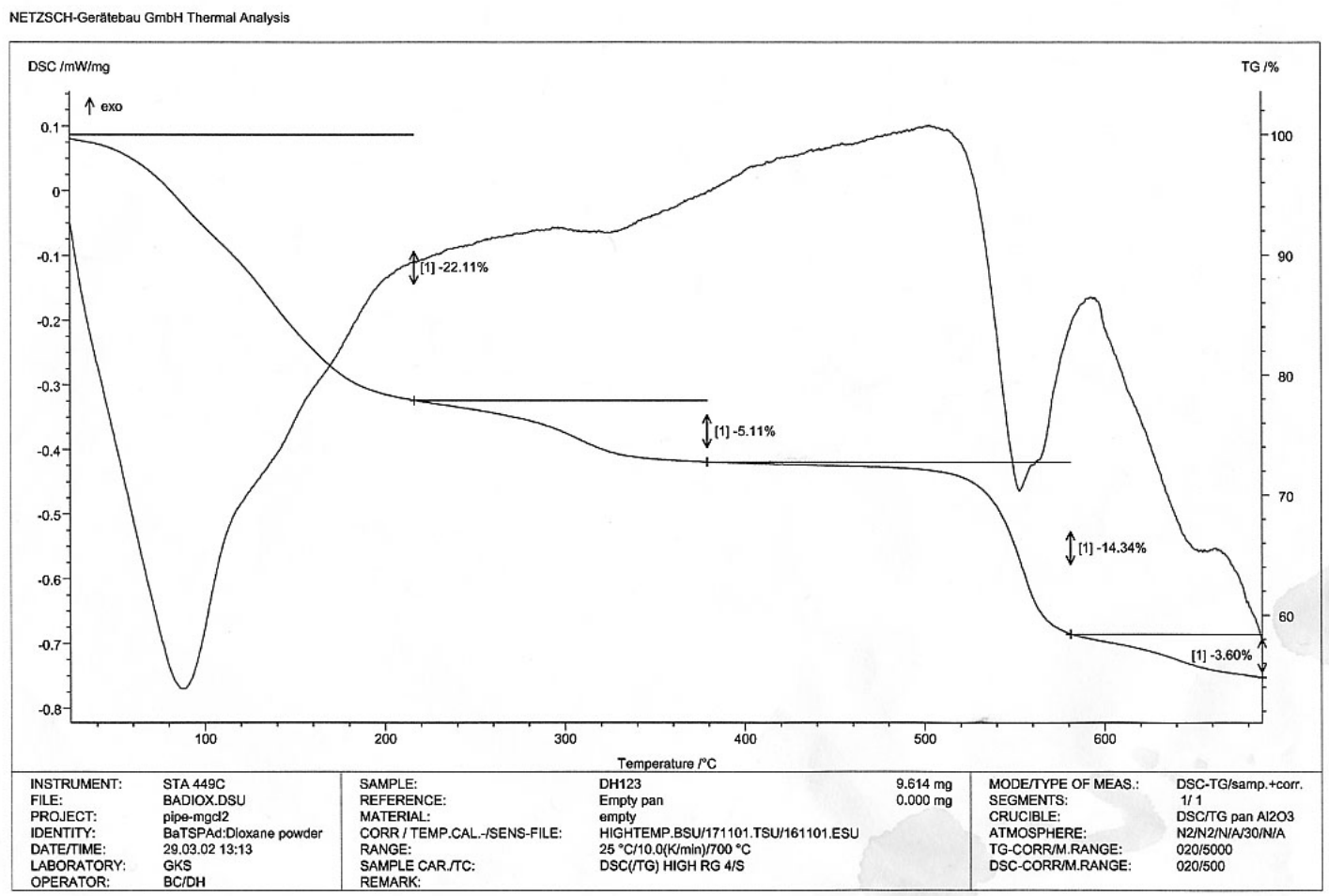


Figure S10: Elemental Analyses for $\left[\mathrm{Ba}_{2}(\mathrm{~L})\left(\mathrm{H}_{2} \mathrm{O}\right)_{4}(\right.$ dioxane $\left.)\right] \bullet\left(\mathrm{H}_{2} \mathrm{O}\right)_{3}(\text { dioxane })_{3}$

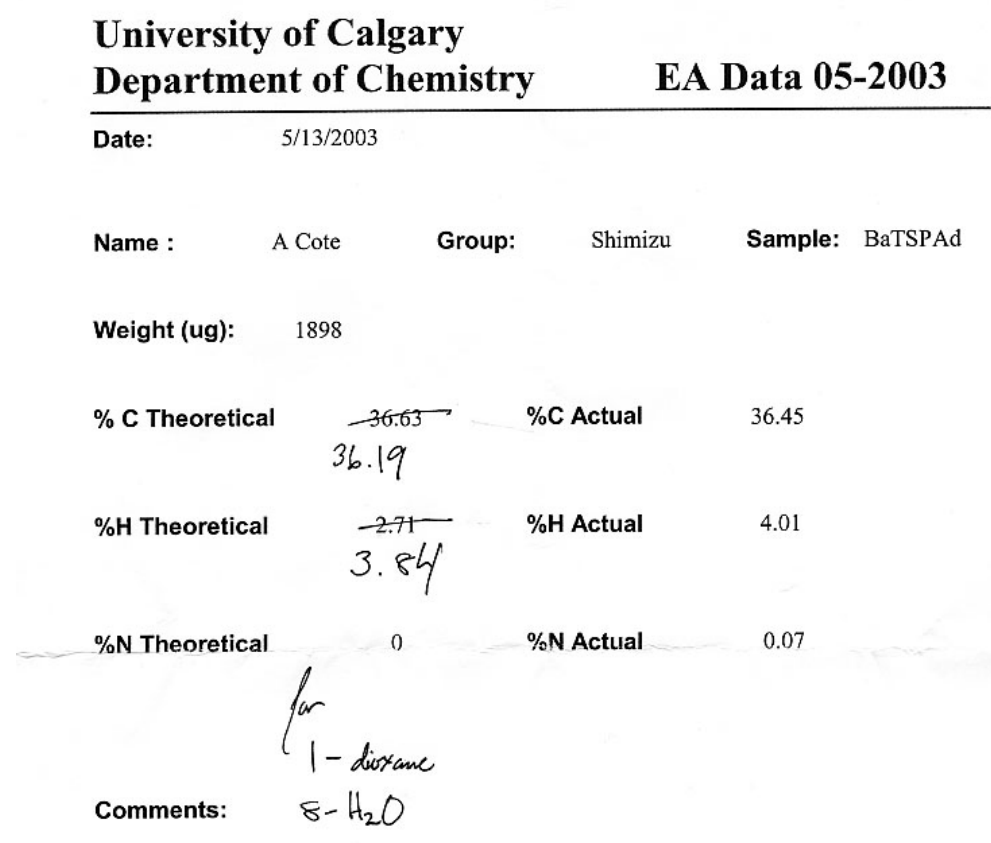

Figure S11: IR Spectrum for $\left[\mathrm{Ba}_{2}(\mathrm{~L})\left(\mathrm{H}_{2} \mathrm{O}\right)_{4}(\right.$ dioxane $\left.)\right] \bullet\left(\mathrm{H}_{2} \mathrm{O}\right)_{3}(\text { dioxane })_{3}$

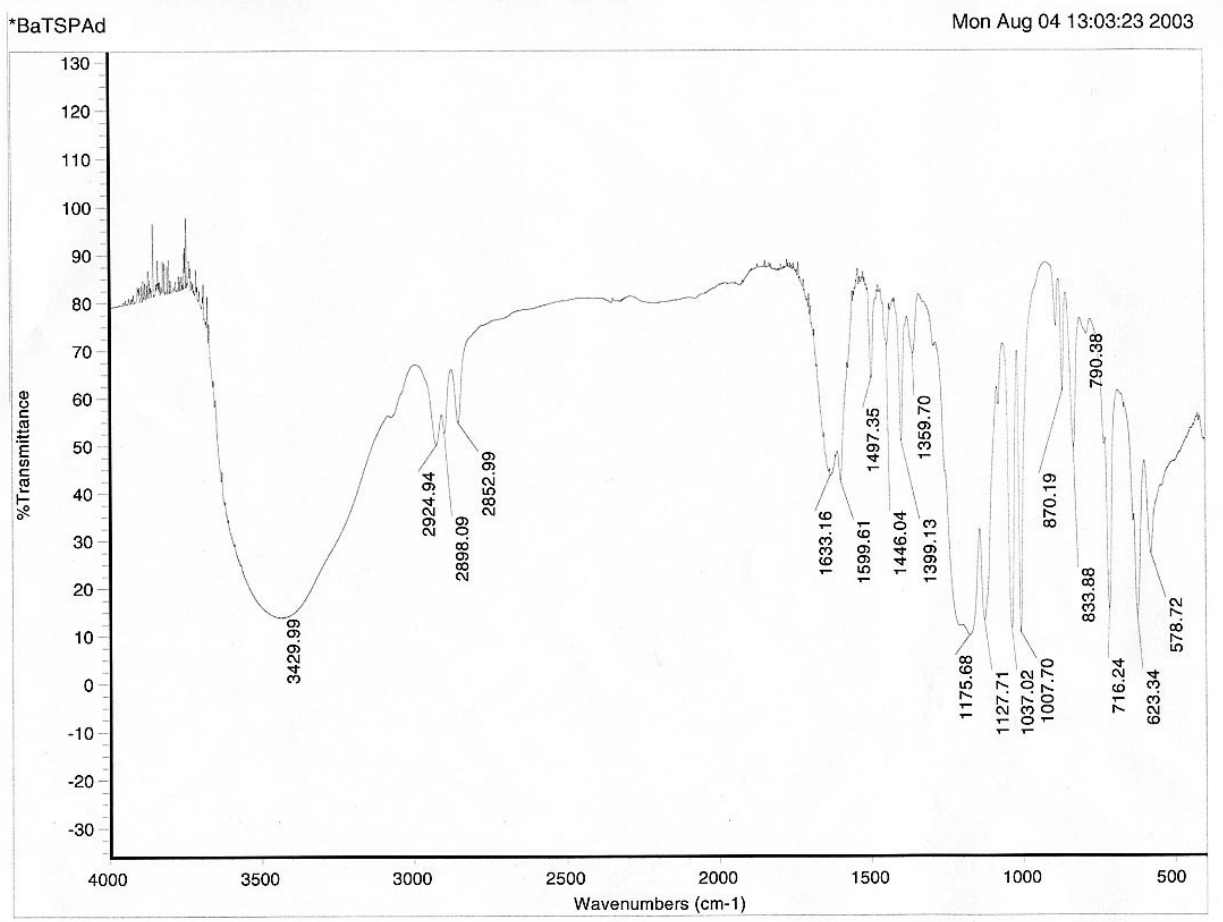

